NATURAL RESOURCE MODELING

Volume 14, Number 2, Summer 2001

\title{
WEIGHT-CONTROLLED RECRUITMENT OF THE ANCHOVY IN THE LATE LARVAL STAGE
}

\author{
OLIVIER PARDO \\ I.P.R.A., ERS 2055 \\ Lab. de Mathématiques Appliquées \\ Université de Pau \\ 64000 Pau, France \\ E-mail: olivier.pardo@univ-pau.fr \\ OVIDE ARINO \\ I.R.D.-GEODES \\ 93143 Bondy, France
}

\begin{abstract}
A formula for the rate of recruitment of the anchovy in the late larval stage is proposed. Recruitment is assumed to occur when the weight crosses a threshold value. The study is undertaken in the framework of a model of fish dynamics which is presented in the first part of the paper.
\end{abstract}

KEY WORDS: Population dynamics, diffusion and advection processes, recruitment.

1. Introduction. The fishing of small clupeids represented $25 \%$ of world tonnage of fish in 1993 with 21 million tons, see Csirke [1995] for more details. But, between the years 60/70, the tonnage was reduced by half. On the whole, between France and Spain, the value of this fishery comes to about US $\$ 80$ millions. More than 400 ships and between 2,000 and 3,000 fishermen are involved. Thus, there exists an increasing economic need for evaluating the abundance of anchovy and its variations.

The anchovy species that we study here is 'Engraulis encrasicolus' of the Bay of Biscay. As with most fish, the life cycle of the anchovy is divided essentially into four distinct stages marked by specific physiological behavioral traits: the egg stage, the larval stage, the juvenile and the adult stages. The first two stages can be subdivided into shorter periods, each of them being associated with the acquisition of a particular organ in the development of the embryo or of a function during the larval growth. Two especially crucial steps in the development are 1) the resorption of the yolk-sac, when the larva switches from endogenous to exogenous feeding (Regner [16]); 2) the period when the swimbladder becomes functional, allowing the larva to control its vertical movement.

Copyright (C)2001 Rocky Mountain Mathematics Consortium 
In this paper we are interested in modeling only the segment of the larval stage which extends from the resorption of the yolk-sac to the beginning of the active vertical movement. During this period, the larvae are still passive. Whereas, during the egg stage and the early larval stage, until the resorption of the yolk-sac, the anchovy is selfsufficient and its mortality is mainly caused by predation, including cannibalism, and natural mortality, this is not so in the next part of the larval stage, after the larva switches to exogenous feeding. This is a period when the larva is the most sensitive to its environment because it must find food to survive. But, its active movement is restricted since the swimbladder is not functional yet, thus, it does not control its vertical movement. Its survival is highly dependent on the presence, in its immediate vicinity, of a sufficient density of phytoplankton, its principal food. At the end of this period, the larva is active and it starts to gather with other anchovies and form fish schools. This is the beginning of the late larval stage.

The main purpose of the work we present here is to describe a model of population dynamics for the anchovy in the last segment of the passive larval stage, from the resorption of the yolk-sac to the beginning of the late larval stage. The model takes into account, in two separate equations, both the demography and the biology. Demography means the number of individuals in terms of time and space; while biology refers to the mechanisms by which the individuals grow and progress through the various stages. We assume that these mechanisms can be globally represented by the weight; that is, we model the variation of weight, at the individual level, as a balance of the phytoplankton eaten, on the one hand, and the cost incurred by larval activity on the other hand. The other purpose of this work, not completed yet, is to estimate the density of larvae arriving in the late larval stage. More precisely, we aim at estimating a probability of recruitment of the post-yolk-sac larvae into the active stage (late larvae) in terms of some parameters and as a function of time. This is similar in spirit to a previous work by Arino et al. [1] on the sole of the Bay of Biscay, Solea solea, where such an estimate was obtained for a much simpler model. What we present here on this subject is only preliminary.

This paper is organized as follows. In the next section we will present the state variables; in Section 3 we will set up the model which will be considered throughout the paper. We determine an equation for each 
of the state variables. Section 4 is devoted to the estimation of the number of larvae entering the active vertical movement. We follow the line of research initiated by Arino et al. [1]; that is, we are looking for somewhat explicit formulae allowing in particular the expression of the late larvae belonging to a particular cohort in terms of the larvae of the same cohort at the end of the yolk-stage. Section 4 consists of two main parts: in the first part, we establish a general formula for the time rate of change of late larvae. In order to keep the computations simple enough, diffusion is neglected. In the second part, two examples are presented in detail. The first example is a slight generalization of the situation considered by Arino et al. [1] for the dynamics of the sole. Example 2 explores the role played by spatial advection in the development of larvae. At the expense of further simplifications, we derive a formula which illuminates the role of space in the recruitment of larvae. The main part ends with a brief discussion. To simplify the discussion in this paper we add an Appendix.

2. State variables. In the sequel we consider the evolution of cohorts of anchovy larvae which are carried by the current in a certain domain $D$ of the ocean, and we consider that $t=0$ stands for the first day of the current year. We consider four main state variables:

$L_{e}(a, t, P)$ is, at time $t$, the density, with respect to age $a$ and position $P$ of individuals in the passive larval phase. The index $e$ below $L$ stands for "early." $P \equiv(x, y, z)$ is a point in the sea: $x$ is the abscissa on a west-east axis, $y$ is the abscissa on a south-north axis and $z$ is the depth, oriented positively towards the surface. The domain $D$ is assumed to be of a size such that the sea surface can be considered a plane. Ideally, $D$ is a region enclosing an isolated patch of eggs, with lateral boundaries far enough not to be reached by the eggs and subsequent material during the time of the observations. The origin is a point in the sea surface, in the "middle" of $D$.

$W_{e}(a, t, P)$ is, at time $t$, the weight density with respect to age and space of individuals in the passive larval phase.

$\varphi(t, P)$ is, at time $t$, the density with respect to space of phytoplankton cells.

$N(t, P)$ is, at time $t$, the concentration of nutrients with respect to space. 


\section{The model equations.}

3.1 The dynamics of the passive larvae density. The function $L_{e}$ satisfies the following system of equations

$$
\begin{aligned}
\frac{\partial L_{e}}{\partial a}+\frac{\partial L_{e}}{\partial t}+\nabla_{P} \cdot\left[L_{e} \vec{V}(t, P)\right] & +\frac{\partial}{\partial z}\left[K(t, z) \frac{\partial L_{e}}{\partial z}\right]=-\mu(a, t) L_{e} \\
L_{e}(a, 0, P) & =0 \\
L_{e}(0, t, P) & =L_{r}(t, P)
\end{aligned}
$$

$\vec{V}(t, P)$ is the velocity vector field of the sea current. $K(t, z)$ is the turbulent diffusion coefficient that depends on time and the depth (Pond and Pickard [1983]).

$\mu(a, t)$ is the mortality rate of the passive larvae at age $a$, due to natural mortality and the effect of predation.

$L_{r}(t, P)$ is the density with respect to $t$ and $P$ of the larvae which have just gone out of the yolk-sac period. The index $r$ is for "resorbed." Integration over a region $\Omega$ of the ocean yields the number of larvae reaching the post yolk-sac stage per unit of time. In this work, $L_{r}$ is data for the problem. The only systematic data for anchovy fish larvae are those collected in egg surveys. Such surveys use sampling devices which can capture very small biotic material: the higher dispersion of larvae compared to eggs makes estimation of larval abundance based on such samples questionable. So, only the eggs at the various stages of their development can be reasonably, and are in fact, estimated. The dynamics of the anchovy, from the time when eggs are laid and fertilized to the end of the yolk-sac period, can be described from a model of larval growth and survival (see for example Arino et al. [1999]). The input of the model by Arino et al. [1996] is the time and space distribution of eggs: size growth of eggs and larvae is modeled by empirical growth functions determined in the laboratory, which, under saturated phytoplankton concentration, depend only on the temperature. This situation suits satisfactorily the early life of the anchovy when it does not yet feed on phytoplankton.

Boundary conditions both at the sea-bed and on the surface, and in the horizontal directions (lateral boundaries) are missing. We point out 
that the lateral boundary conditions can be omitted since horizontal migration is a transport with finite speed and we are assuming that we start from a patch of eggs which needs more time to reach those boundaries than the time of observation. This assumption is not essential, however, and a more general situation could be handled. The important fact here is that we are not taking into account the larvae that enter the domain $D$, from outside, after $t=0$. The vertical boundary conditions should express the fact that both the surface and the seabed are barriers that none of the materials considered in this model (larva, phytoplankton, nutrients) can cross. Vertical boundary conditions are only useful in dealing with the second order diffusion terms which, for simplicity, we will drop in our study.

3.2 The dynamics of the passive larvae weight. The function $W_{e}$ satisfies the following system of equations

$$
\begin{gathered}
\frac{\partial W_{e}}{\partial a}+\frac{\partial W_{e}}{\partial t}+\nabla_{P} \cdot\left[W_{e} \vec{V}(t, P)\right]+\frac{\partial}{\partial z}\left[K(t, z) \frac{\partial W_{e}}{\partial z}\right] \\
=k(t) f\left(t, P, T(t, .), W_{e}, \varphi\right) \varphi-\left(\alpha+\beta\left(w^{*}-W_{e}\right)^{\theta}\right) W_{e} \\
W_{e}(a, 0, P)=0 \\
W_{e}(0, t, P)=h(t, P)
\end{gathered}
$$

Here $k(t)$ is a periodic function which models the feeding regime of the larvae within a 24 hour period. For example, $k \equiv 0$ during the night, since the larvae do not regularly feed at night, see Re [1994] for more details. At the same time, the larvae possess an absorption capacity $f\left(t, P, T(t,),. W_{e}, \varphi\right)$, which we assume to increase with weight and to be nonlinear. The dependence of $f$ on $\varphi$ is more of a threshold nature: the dependence of $f$ on $\varphi$ is such that $f=0$ if $\varphi$ is less than some threshold value (below a certain density, it is likely that the rate of capture of the phytoplankton by the larvae will be close to zero). We assume a functional dependence of $f$ on the temperature. The larvae lose weight for one main reason, the energy consumption by the larval metabolism. The model chosen for this takes into account the observed fact that life is more difficult for underweight larvae than it is for those whose weight is close to the threshold for reaching the juvenile stage. Worsening of life condition, as a result of poor feeding, 
is twofold: 1) it costs more energy for a larva to sustain its activity when it is underweight, which is stressed by the quantity $\beta\left(w^{*}-\right.$ $\left.\left.W_{e}\right)^{\theta}, \theta>0 ; 2\right)$ such larvae get less food than those with larger weights, which is accounted for by the monotonicity of $f\left(t, P, T(t,),. W_{e}, \varphi\right)$. The way food deprivation acts on the feeding capacity is, however, more complicated than the above point 2) suggests. A more sound proposition would be that larvae deprived of food for some days will find it more difficult to successfully catch the prey. So, the rate of successful encounter of the prey does not just depend on the actual weight of the larva, it should reflect cumulative loss over some period of time. The dependence of $f$ on $t$ and $P$ gives the possibility of taking into account the effect of any admissible abiotic factors we can think of. For example, this can be the accessibility of the phytoplankton, which certainly depends on the physical environment of the larvae, or it can be the temperature, as a global indicator of this environment. Finally, we note that $w^{*}$ is the critical weight that passive larvae have to reach to cross the passive phase and the function $h(t, P)$ is the weight of larvae at the beginning of the exogenous feeding phase, that is to say, the average weight of the larvae which at time $t$ are in $P$ and start to feed on phytoplankton.

3.2.1 Remark: weight versus size. We choose the weight as a structure variable. In the literature, however, the body size, that is, the length of the body of an individual, is used, rather than the weight, in particular associated with the completion of physiological stages: on the body size scale, the late larval stage starts when the larva is around $10 \mathrm{~mm}$ long (Peterman et al. [1988]). This is roughly when the larva starts to control its vertical movement (functional swimbladder) (Mullin [1993], Fig. 4.9). There are allometric relationships between the size and the weight. In terms of such relationships, one can consider the weight and the body size as equivalent parameters and, in principle, indifferent to one or the other. It is intuitively clear however that there must be some fundamental differences between the two quantities. On the one hand, the weight seems to be more sensitive, especially during the larval growth, to the daily food regime, while the body length changes at a slower pace. It is more suited than length to daily bookkeeping. On the other hand, the respective variabilities of the weight and the length on completion of developmental stages have been compared: it is higher 
for the weight. So, the length should be preferred to the weight when setting up threshold values for significant physiological changes. But, no clear law of variation of the length is known. This explains that, in spite of its shortcomings, we are using the weight in this paper, and we use $w^{*}$ as a threshold value for the completion of the passive larval stage.

3.3 The dynamics of phytoplankton cells and nutrient. The functions $\varphi$ and $N$ satisfy the following system of equations

$$
\frac{\partial \varphi}{\partial t}=[\alpha(I) \Phi(N)-m] \varphi-\nabla_{P} \cdot[\vec{V}(t, P) \varphi]+\frac{\partial}{\partial z}\left[K(z, t) \frac{\partial \varphi}{\partial z}\right]
$$

$$
\begin{aligned}
\frac{\partial N}{\partial t}=-\gamma[\alpha(I) \Phi(N)-\varepsilon m] \varphi & -\nabla_{P} \cdot[\vec{V}(t, P) N]+\frac{\partial}{\partial z}\left[K(z, t) \frac{\partial N}{\partial z}\right] \\
\varphi(0, P) & =\varphi_{0}(P) \\
N(0, P) & =N_{0}(P)
\end{aligned}
$$

The specific growth rate for excess nutrient, $\alpha(I)$, was obtained from the light intensity $I$ at any depth by the 'tanh' formula of Jassby and Platt [1976]

$$
\alpha(I)=\alpha_{0} P_{m} \tanh \left(\frac{\alpha^{\prime} I}{P_{m}}\right),
$$

where $\alpha^{\prime}$ is the initial slope of the light saturation curve and $P_{m}$ is the assimilation number, $\alpha_{0}$ is a constant. The extent of reduction in growth rate by nutrient limitation is determined by a Michaelis-Menten relation

$$
\Phi(N)=\frac{N}{N+\varpi},
$$

where $\varpi$ is the half-saturation, independent of depth, see MacIsaac and Dugdale [1969] for more details. The specific loss rate, $m$, includes the mortality of cells due to death, grazing and loss due to respiration. This death regenerates the nutrient with an efficiency $\varepsilon, \varepsilon \in[0,1]$. Assuming the nutrient to be nitrogen, $\gamma$ is the conversion factor. The role of the anchovy in depleting the phytoplankton is neglected, as it can be noticed that equation (3.7) does not contain any of the 
state variables associated with the anchovy. However, equation (3.4) indicates that the larvae eat phytoplankton, and as a consequence of the book-keeping principle, the nonlinear term $k(t) f\left(W_{e}\right) \varphi$ should be added to equation (3.7) with a negative sign. Accordingly, some part of the larval biomass should be added to equation (3.8). All this is considered negligible compared to the other factors acting on the phytoplankton. In fact, rather than a prey-predator relationship, with variations on each species induced by the other species, the coupling between the phytoplankton and the larvae should be thought of as one between the parameters and the state variables of a system: the phytoplankton, ideally at a steady state, would be seen as a driving function entering the equations of the larvae, with possibly a threshold value $\varphi^{*}$ such that, in the regions where $\varphi<\varphi^{*}$ or the time periods when $\varphi<\varphi^{*}$, the feeding rate of the larvae will be so low that it will drive the larvae to extinction. In fact, there should be a family of such steady-states, dependent upon the values of some external parameters (wind, temperature, light), some solutions being uniformly above $\varphi^{*}$, some uniformly below $\varphi^{*}$, and some crossing the value $\varphi^{*}$. Finally, some sort of stability should hold so that transient solutions arising from perturbations of the external parameters would rapidly approach one of the steady-states after the perturbations have faded. Verifying rigorously such a scenario is a vast mathematical program which is out of the scope of this paper.

If we suppose that $\varphi \equiv \varphi(t, z)$ and that $\vec{V}(t, P)$ is a constant vector, then we get the model proposed by Taylor et al. [1986]. We have performed an analytical study of this model, see Pardo [2000] for more details.

3.4 The complete system. The complete system is composed of the four equations, (3.1), (3.4), (3.7) and (3.8) satisfied respectively by $L_{e}, W_{e}, \varphi$ and $N$, and the initial and boundary conditions, (3.2) and (3.3) for $L_{e},(3.5)$ and (3.6) for $W_{e}$ and (3.9) and (3.10) for $\varphi$ and $N$.

We will not deal here with the solution of the system. We will now elaborate on the computation of a probability of recruitment in the late larval stage. In order to keep the computations simple enough, we assume from now on that diffusion is negligible, so that all the equations reduce to transport equations and can be solved using the method of characteristic lines. 
3.5 The solution of the equation of larvae. In the sequel we make the hypothesis of incompressibility (Pond and Pickard 1983]), that is to say,

$$
\operatorname{div} \vec{V}(t, P)=0 .
$$

Thus, equation (3.1) can be rewritten as

$$
\frac{\partial L_{e}}{\partial a}+\frac{\partial L_{e}}{\partial t}+\vec{V}(t, P) \cdot \vec{\nabla}_{P} L_{e}=-\mu(a, t) L_{e} .
$$

We reduce the problem to one time variable (instead of $a, t, P$ ) by restricting equation (3.1) along the characteristic lines of the equation, that is, the trajectories of the solutions of the system of ordinary differential equations

$$
\left\{\begin{array}{l}
\frac{d a}{d s}=1 \\
\frac{d t}{d s}=1 \\
\frac{d P}{d s}=\vec{V}(t(s), P(s)) .
\end{array}\right.
$$

Since $L_{e}(a, 0, P)=0, L_{e}$ remains identically null along the characteristic lines initiated from the half line $t=0, a>0$. Only the characteristic lines starting from $a=0, t>0$ are of interest here. The general expression of such a solution is

$$
a=s, \quad t=s+t_{0}, \quad P=\bar{P}(s)=\bar{P}\left(s, t_{0}, P_{0}\right)
$$

where $\bar{P}\left(s, t_{0}, P_{0}\right)$ is the position at time $t$ of a particle whose initial position at time $t_{0}$ was $P_{0}$. Later on in the text, we will also use the flow in the coordinates $(t, P)$ associated with equation (3.12), that is,

$$
\Phi\left(a, t_{0}, P_{0}\right)=\left(t_{0}+a, \bar{P}\left(a, t_{0}, P_{0}\right)\right) .
$$

We denote accordingly,

$$
\begin{aligned}
\bar{L}_{e}(s) & =L_{e}\left(s, s+t_{0}, \bar{P}\left(s, t_{0}, P_{0}\right)\right), \\
\bar{\mu}(s) & =\mu\left(s, s+t_{0}\right),
\end{aligned}
$$

and, similarly, for the restriction to a characteristic line of any function of the variables $a, t, P$. 
The equation satisfied by $\bar{L}_{e}$ is

$$
\frac{d \bar{L}_{e}}{d s}=-\bar{\mu}(s) \bar{L}_{e}(s)
$$

This equation has for a solution

$$
\bar{L}_{e}(s)=\bar{L}_{e}(0) \exp -\int_{0}^{s} \bar{\mu}(\sigma) d \sigma .
$$

We have to determine $\bar{L}_{e}(0)=L_{e}\left(0, t_{0}, P_{0}\right)$. In view of (3.3), we have

$$
L_{e}\left(0, t_{0}, P_{0}\right)=L_{r}\left(t_{0}, P_{0}\right)
$$

thus,

$$
\bar{L}_{e}(s)=L_{r}\left(t_{0}, P_{0}\right) \exp -\int_{0}^{s} \bar{\mu}(\sigma) d \sigma .
$$

Coming back to the expression of $L_{e}$, we obtain the following

$$
L_{e}(a, t, P)=L_{r}(t-a, \bar{P}(t-a, t, P)) \exp -\int_{0}^{a} \mu(\sigma, t-a+\sigma) d \sigma
$$

\section{Estimate of the number of larvae entering the late larval} stage. We start with a brief discussion about the way we want to compute an estimate of the number of larvae entering the late larval stage. The method we use is based on the same principle as the one used in a previous paper by Arino et al. [1996] for estimating a probability of recruitment of the sole, Solea solea, in the juvenile stage. The computations in that case were very simple, so we will quickly go through them in order to explain what we want to do here. First of all, we underline a distinctive feature of the model under consideration, also present in Arino et al. [1996], the weight is treated as another state variable of the population, together with the population density, that is to say, each cohort is represented, at each time $t$ by the pair $\left(L_{e}(a, t, P), W_{e}(a, t, P)\right)$. Alternatively, one could have considered the weight as another structure variable of the population density. The main role of the weight function in the context of this section is to allow the computation of the time, age and position at which individuals exit 
from the larval stage. In Arino et al. [1996], it was assumed that the instantaneous rate of variation of the weight is a function $g(t, T)$ of the average temperature and time, $t$, only, not of the space and the actual weight:

$$
\frac{\partial W_{e}}{\partial a}+\frac{\partial W_{e}}{\partial t}=g(t, T(t)) .
$$

We point out that equation (4.1) can be obtained as a special case of equation (3.4), under a number of assumptions that we are not going to detail here. Such a simple model can be integrated easily and yields the following expression for $W_{e}$, neglecting the weight at age $a=0$,

$$
W_{e}(a, t)=\int_{t-a}^{t} g(s, T(s)) d s .
$$

Assuming that $g$ is positive, one obtains immediately that $W_{e}(a, t)$ is increasing in $a$, so that the equation

$$
W_{e}(a, t)=w^{*}
$$

has at most one solution. Assuming that there is one for each $t$, we denote it $a^{*}(t)$. The interpretation of $a^{*}(t)$ is that it is the age of those larvae that cross the threshold associated with the weight $w^{*}$ at time $t$, and $t-a^{*}(t)$ is the birth date. Another property that can be deduced from $(4.2)$ is that $a^{* \prime}(t)<1$. This property has a natural interpretation, that is, it means that there is no overlap of successive cohorts at the point of exit from the larval stage: the first individuals to be born will be the first to be recruited. In terms of $a^{*}$ it is possible to express the exit flow rate as a function of the inflow rate: the ratio of the first number to the second one gives the rate of recruitment of the corresponding cohort. A lower estimate of this rate, independent of the cohorts, was computed, which was taken as the probability of recruitment. Here, we follow the same general principles although the situation is a little bit more complicated since the weight is not monotonically increasing. The lack of monotonicity may be due to two causes (see equation (3.4)): the lack of phytoplankton or the excess of energy cost. For a moment, let us come back to the model of growth of Arino et al. [1996], modified by introducing an energy cost of the same type as in equation (3.4). Equation (4.1) becomes

$$
\frac{\partial W_{e}}{\partial a}+\frac{\partial W_{e}}{\partial t}=g(t, T(t))-W_{e}(a, t) \gamma\left(W_{e}(a, t)\right) .
$$


We may again observe that (4.3) is a special case of equation (3.4), providing we assume that in equation (3.4) we can give the dependence on $W_{e}$ in the righthand side a more general form than it has. It is not simple enough, however, to be computed. Assuming for making it even simpler that $\gamma\left(W_{e}\right)$ is a constant $\alpha>0$, equation (4.3) reduces to

$$
\frac{\partial W_{e}}{\partial a}+\frac{\partial W_{e}}{\partial t}=g(t, T(t))-\alpha W_{e}(a, t) .
$$

Let us remember that we are concerned here with the segment of the larval life which goes from the moment when the yolk-sac is resorbed (the beginning of the exogenous feeding), taken as age $a=0$, to the moment when the larva reaches the weight $w^{*}$. We assume that the weight of the larvae at the beginning of exogenous feeding is the same for all larvae, equal to $W_{0}, W_{0}<w^{*}$. Equation (4.4) is to be solved for each age cohort in the post yolk-sac period, that is, individuals having started this period at the same time $t_{0}$. For such a cohort, there is the following relationship between the age and the chronological time: $t=t_{0}+a$. Defining

$$
\bar{W}_{e}(a)=W_{e}\left(a, t_{0}+a\right),
$$

equation (4.4) yields the following

$$
\bar{W}_{e}^{\prime}(a)=\bar{g}(a)-\alpha \bar{W}_{e}(a),
$$

where $\bar{g}(a)=g\left(t_{0}+a, T\left(t_{0}+a\right)\right)$. We also have: $\bar{W}_{e}(0)=W_{0}$. The solution reads as

$$
\bar{W}_{e}(a)=\exp (-\alpha a) W_{0}+\int_{0}^{a} \exp (-\alpha(a-s)) \bar{g}(s) d s
$$

which, in terms of the variables $a$ and $t$, gives

$$
W_{e}(a, t)=\exp (-\alpha a) W_{0}+\int_{t-a}^{t} \exp (-\alpha(t-s)) g(s, T(s)) d s .
$$

Comparing with equations (4.1) and (4.2), one can see that $\bar{W}_{e}(a)$ is always increasing in the case of equation (4.1), while equation (4.5) shows that the same property holds here only if $\bar{g}(a)>\alpha \bar{W}_{e}(a)$, 
which has the obvious interpretation that the weight gained from food consumption is more than that lost in sustaining activity. We also noticed that $W_{e}(a, t)$ is increasing in $a$, in the case of equation (4.1); this ensures that at most one cohort reaches the juvenile stage at a given time. This is not always true in the case of equation (4.5). Taking the partial derivative, with respect to $a$, of formula (4.6) leads to

$$
\frac{\partial W_{e}(a, t)}{\partial a}=\exp (-\alpha a)\left[-\alpha W_{0}+g(t-a, T(t-a))\right] .
$$

Formula (4.7) shows that $\left(\partial W_{e}(a, t) / \partial a\right)$ has a constant sign along the trajectory of a cohort determined by the feeding conditions which were prevailing at the beginning of the trajectory (age $a=0$ ). It is difficult to interpret this property since it is based at least in part on the technical assumption that the initial weight is the same for all the cohorts. It suggests incorporating another threshold in the model, that is, only the larvae whose endogenous feeding stage ends at a time $t$ such that

$$
g(t, T(t)) \geq \alpha W_{0}
$$

will switch to active feeding at this time. The other ones reach the value $W_{0}$ at a time $t_{1}$ such that

$$
g\left(t_{1}, T\left(t_{1}\right)\right)<\alpha W_{0} .
$$

No feeding occurs from this time on, so these larvae start to lose weight exponentially, that is, $W_{e}(t)=W_{0} \exp \left(-\alpha\left(t-t_{1}\right)\right)$, until either the weight goes below a certain value $w_{\min }$, a threshold for starvation and death, whatever future feeding conditions could be, or $t_{2}>t_{1}$ exists such that

$$
g\left(t_{2}, T\left(t_{2}\right)\right) \geq \alpha W_{e}\left(t_{2}\right) \geq \alpha w_{\min } .
$$

Active feeding will then start from the very first moment $t_{2}$ when the above two inequalities hold simultaneously. The weight starts to grow, from $t_{2}$ onwards, according to the same formula as (4.6) with $W_{0}$ changed to $W_{e}\left(t_{2}\right)$. Age in active feeding should be initialized to 0 at this time.

The condition $g(t, T(t)) \geq \alpha W_{0}$ to be satisfied at the beginning of the active feeding period would be a rough way to account for the accessibility of the prey, in particular at a time when the larva has 
not yet adapted to its new feeding habit. Accessibility of the resource is a concept which combines several factors: the concentration of the resource (here, the phytoplankton), its quality (some prey will be too big, others could be too small), as well as the physical environment (current and turbulence). All these factors together make the resource more or less available. This degree of availability compares to the cost incurred by the larva in its effort to catch the prey.

The mechanisms involved in the successful feeding of some larvae and the failure of probably many others are far from being understood yet. There is a growing view that larval survival is tightly related with them aggregating around high concentrations of food. This view is in particular supported by laboratory experiments which indicate that the minimum rate of phytoplankton necessary to sustain larval life in the laboratory is well above the usual concentrations of phytoplankton observed in the field. It is also believed that such findings should be made as soon as possible after the resorption of the yolk-sac. Finally, so to speak, success attracts success and accordingly for failure: larvae that were able to feed abundantly immediately after they started to look for food will have the possibility of going through periods of low food abundance. At the other end, those larvae which were not successful from the beginning will have more and more difficulty to search. The effect of entrainment produced by the first feeding success is roughly reflected by condition (4.8). There are however probably many other circumstances in its active feeding life time when the larva is faced with low food accessibility: its ability to withstand such conditions will then increase with weight (as modeled in equation (3.4)). A deterministic description of the succession of bad and good feeding periods and an estimation of how bad they can be is out of reach. A very preliminary step in this direction is the choice of the value $w_{\min }<W_{0}$ such that no larva, which, after having reached the weight $W_{0}$, falls below $w_{\min }$, will become juvenile.

We now return to equation (3.4) where, as already mentioned, we assume that $K(t, z)=0$, and we assume that the quantities $W_{0}, w^{*}$ and $w_{\min }$ are as in the previous discussion. If $W_{e}(a, t, P)$ is a solution of (3.4), with $W_{e}\left(0, t_{0}, P_{0}\right)=W_{0}$, and $\bar{W}_{e}(a)$ is the restriction of $W_{e}(a, t, P)$ to a characteristic line starting from $\left(0, t_{0}, P_{0}\right)$, then we follow $\bar{W}_{e}(a)$ until the first $\bar{a}=\bar{a}\left(t_{0}, P_{0}\right)>0$ such that

$$
\bar{W}_{e}(\bar{a})=w^{*}
$$


providing that $\bar{W}_{e}(a)>w_{\min }$ for every $0<a<\bar{a}$. In order to compute the flux of larvae entering the late larval stage, it is first necessary to determine the set of points (the admissible set) in the space of $(a, P)$ where, at time $t, W_{e}(a, t, P)=w^{*}$. Let $(a, t, P)$ be a given point. In terms of the flow $\Phi$ defined by (3.13), we have

$$
\left(t_{0}, P_{0}\right)=\Phi(-a, t, P) ;
$$

therefore, the characteristic line passing through $(a, t, P)$ is given by

$$
(t(s), P(s))=\Phi(s-a, t, P) .
$$

So, the relations defining the exit set at time $t, \mathcal{R}(t)$ is

$$
\begin{gathered}
W_{e}(a, t, P)=w^{*} \text { and } \\
w_{\min }<W_{e}(s, \Phi(s-a, t, P))<w^{*} \text { for } 0<s<a \\
\mathcal{R}(t)=\{(a, P): \text { formula (4.9) holds }\}
\end{gathered}
$$

From (4.9), it is clear that to a given $(t, P)$ is associated with at most one $a$ such that (4.9) holds. Assuming for simplicity that there is always a solution, then we can define $a^{*}(t, P)=a$, with $a$ given by (4.9). The exit set $\mathcal{R}(t)$ is the boundary of a larger open set, which we will call the admissible set, denoted $\mathcal{A}(t)$ and defined as follows

$$
\begin{aligned}
\mathcal{A}(t)=\{ & (a, P): W_{e}(a, t, P)<w^{*}, \text { and } \\
& \left.w_{\min }<W_{e}(s, \Phi(s-a, t, P))<w^{*} \text { for } 0<s<a\right\} .
\end{aligned}
$$

We point out that for each $(a, P) \in \mathcal{A}(t)$, we have $a<a^{*}(t, P)$. The section of $\mathcal{A}(t)$ over a point $P$ is the collection of ages of larvae, all in between the post yolk-sac and the functional swimbladder period, susceptible to reach the juvenile stage. We denote

$$
\mathcal{A}_{\sigma}(t)=\{P: \exists a,(a, P) \in \mathcal{A}(t)\} .
$$

For each point $P$ of the set $\mathcal{A}_{\sigma}(t)$, we determine all the trajectories that can be followed by larvae, which at time $t$ are positioned at $P$, and have never experienced starving conditions. For each $P \in \mathcal{A}_{\sigma}(t)$, the set of $a$, such that $(a, P) \in \mathcal{A}(t)$ is a union of open intervals: each "hole" between two intervals corresponds to a trajectory crossing an 
inhospitable region where larvae would be deprived of food to the point of going below the threshold for starvation.

We will now evaluate the variation, within a small time interval $[t$, $t+\Delta t$, of the population of larvae, located in a given region $\Omega$ of the ocean, which have the potential to reach the late larval stage. At time $t$, the number of larvae in this situation is given by the following integral

$$
L_{\text {pot }}(t)=\int_{\mathcal{A}_{\Omega}(t)} L_{e}(a, t, P) d a d P
$$

where

$$
\mathcal{A}_{\Omega}(t)=\{(a, P):(a, P) \in \mathcal{A}(t) \text { and } P \in \Omega\} .
$$

We denote $L_{l}(t, P)$ the spatial density of the larvae entering the late larval stage at time $t$. The variation of $L_{\text {pot }}(t)$ between $t$ and $t+\Delta t$ is caused by four factors: 1) The arrival of new post yolk-sac larvae, of weight $\left.W_{0} ; 2\right)$ The natural mortality; 3) The advection by currents; 4) The passage into the late larval stage.

New post yolk-sac larvae:

$$
\left[\int_{\Omega} L_{e}(0, t, P) d P\right] \Delta t
$$

\section{Natural mortality:}

$$
\left[\int_{\mathcal{A}_{\Omega}(t)} L_{e}(a, t, P) \mu(a, t) d a d P\right] \Delta t
$$

\section{Advection by currents:}

$$
\left[\int_{\partial \mathcal{A}_{\Omega \sigma}(t)}\left[\int_{\{a:(a, P) \in \overline{\mathcal{A}(t)}\}} L_{e}(a, t, P) d a\right] \vec{V}(t, P) \cdot \vec{n}(P) \sigma(d P)\right] \Delta t
$$

in which the notation $\partial \mathcal{A}_{\Omega \sigma}(t)$ corresponds to the boundary of $\mathcal{A}_{\Omega \sigma}(t)$, $\overline{\mathcal{A}(t)}$ is the closure of $\mathcal{A}(t), \vec{n}(P)$ is the unit normal vector to $\partial \mathcal{A}_{\Omega \sigma}(t)$ 
at the point $P$, oriented outwards and $\sigma(d P)$ is the element of surface on $\partial \mathcal{A}_{\Omega \sigma}(t)$.

Passage into the late larval stage. We obtain the following general formula for the flux of early juveniles

$$
\begin{aligned}
L_{l}(t, P)= & 1_{\mathcal{A}_{\sigma}(t)}(P) L_{e}\left(a^{*}(t, P), t, P\right) \\
& -1_{\mathcal{A}_{\sigma}(t)}(P)\left[\int \vec{\nabla}_{P} 1_{\{a:(a, P) \in \overline{\mathcal{A}(t)\}}}(a) L_{e}(a, t, P) d a\right] \\
& \cdot \vec{V}(t, P)+1_{\Sigma_{\sigma}(t)}(P) L_{e}\left(a^{*}(t, P), t, P\right) \frac{\left(\partial W_{e} / \partial t\right)}{\left|\left(\partial W_{e} / \partial a\right)\right|}
\end{aligned}
$$

see Appendix, Section 1, for more details. We will now illustrate formula (4.15) by two examples.

4.1 Example 1. We consider the situation considered in Arino et al. [1996], that is, growth is governed by equation (4.1), which integrates to

$$
W_{e}(a, t)=W_{0}+\int_{t-a}^{t} g(s, T(s)) d s .
$$

$W_{e}$ is increasing along each cohort, which implies that $W_{e} \geq W_{0}$. So, $\mathcal{A}(t)$ has a simple description: in particular, $a^{*}(t, P)=a^{*}(t)$ and

$$
\mathcal{A}(t)=] 0, a^{*}(t)[\times D
$$

which yields

$$
\mathcal{A}_{\sigma}(t)=\Sigma_{\sigma}(t)=D \text {. }
$$

Since $\mathcal{A}(t)$ is a product of two sets, with the end points of the set of ages independent of the position, it implies that $\vec{\nabla}_{P} 1_{\{a:(a, P) \in \overline{\mathcal{A}(t)}\}}(a)=0$, formula (5.14) boils down to

$$
L_{l}(t, P)=L_{e}\left(a^{*}(t), t, P\right)+L_{e}\left(a^{*}(t), t, P\right) \frac{\left(\partial W_{e} / \partial t\right)}{\left|\left(\partial W_{e} / \partial a\right)\right|} .
$$

Using the notation $\bar{g}(t)=g(t, T(t))$, we have

$$
\begin{aligned}
\frac{\partial W_{e}}{\partial t} & =\bar{g}(t)-\bar{g}(t-a), \\
\frac{\partial W_{e}}{\partial a} & =\bar{g}(t-a) .
\end{aligned}
$$


Introducing these values in the formula for $L_{l}(t, P)$, we obtain

$$
L_{l}(t, P)=L_{e}\left(a^{*}(t), t, P\right) \frac{\bar{g}(t)}{\bar{g}\left(t-a^{*}(t)\right)} .
$$

Now, differentiating the identity

$$
W_{e}\left(a^{*}(t), t\right)=w^{*}
$$

with respect to $t$ yields

$$
a^{* \prime}(t)=-\frac{\left(\partial W_{e} / \partial t\right)}{\left(\partial W_{e} / \partial a\right)}
$$

In terms of $a^{*}(t)$, formula (4.16) gives

$$
L_{l}(t, P)=\left(1-a^{* \prime}(t)\right) L_{e}\left(a^{*}(t), t, P\right)
$$

which is precisely the formula obtained in Arino et al. [1996] for the sole. Using the expression of $L_{e}(a, t, P)$ in terms of $L_{r}$ given in (3.14), we obtain

$$
\begin{aligned}
L_{l}(t, P)=\left(1-a^{* \prime}(t)\right) \exp - & {\left[\int_{0}^{a^{*}(t)} \mu\left(\sigma, t-a^{*}(t)+\sigma\right) d \sigma\right] } \\
& \cdot L_{r}\left(t-a^{*}(t), \bar{P}\left(t-a^{*}(t), t, P\right)\right) .
\end{aligned}
$$

Formula (4.18) gives the flux of larvae entering the late larval stage at time $t$ and position $P$ in terms of the flux of larvae at the end of the yolk-sac stage, which, at time $t$ and position $P$, would be susceptible to enter the late larval stage. So, the ratio

$$
\frac{L_{l}(t, P)}{L_{r}\left(t-a^{*}(t), \bar{P}\left(t-a^{*}(t), t, P\right)\right)}
$$

gives, at this time and this position, the rate of recruitment of a cohort of larvae at the end of the yolk-sac stage in the late larval stage. In this simplified example where we assume that the growth conditions are the same everywhere, the rate of recruitment is independent of the position; it is a function of time only. On the other hand, the magnitude of the flux depends on the position since the ratio compares the number of 
late larvae at a given position $P$ to the number of larvae that were driven by the current from their previous position $\bar{P}$ where they were at time $t-a^{*}(t)$.

4.2 Example 2. In this example we assume that some spatial dependence holds for weight growth. Our main assumption is that the current is constant and is oriented westwards

$$
\vec{V}(t, P)=\vec{U}=\left(U_{1}, 0,0\right) .
$$

This implies that all the properties under consideration in this study are invariant through time along the north-south and the vertical components. We also assume that the nutrient concentration is constant $N=\bar{N}$, which is feasible under the assumption of velocity constancy (see equation (3.8)). This gives the equation for phytoplankton (equation (3.7)) the following expression

$$
\frac{\partial \varphi}{\partial t}=C(\bar{N}) \varphi-U_{1} \frac{\partial \varphi}{\partial x}
$$

in which $C(\bar{N})$ is the constant rate of production of phytoplankton due to the nutrients. We complete the equation for the phytoplankton by an initial value

$$
\varphi(0, x)=\varphi_{0}(x)
$$

and a boundary condition, which corresponds to a source term

$$
\varphi(t, \xi)=\widetilde{\varphi}(t) .
$$

$\xi$ represents the most coastward abscissa of the region under consideration. Finally, we assume that the growth rate of the weight (equation (3.4) ) as a result of eating phytoplankton is linear in $\varphi$, independent of $W_{e}$, which gives the following equation for the weight

$$
\frac{\partial W_{e}}{\partial a}+\frac{\partial W_{e}}{\partial t}+U_{1} \frac{\partial W_{e}}{\partial x}=k(t) f(t) \varphi-\alpha W_{e} .
$$

It is then possible to integrate the state variables $\varphi, L_{e}$ and $W_{e}$ with respect to the spatial variables $y$ and $z$ and work with the integrated 
state variables, functions of $a, t$ and $x$ only. Thus we obtain (see Appendix, Section 2, for more details),

$$
\begin{cases}L_{l}(t, x) & \\ =\left[1-U_{1} a^{(2) \prime}(x)\right] L_{e}\left(a^{(2)}(x), t, x\right) & \text { if } x>U_{1} t+\xi \text { and } a^{(2)}(x)<+\infty \\ L_{l}(t, x)=0 & \text { otherwise. }\end{cases}
$$

At this point, it would be possible to continue as has been done in Example 1: one can express $L_{e}$ in terms of $L_{r}$ and compute the ratio of the flux of late larvae to the flux of the larvae at the end of the yolk-sac stage. We defer this to future work.

5. Discussion. We have described by mathematical equations the last segment of the passive larval stage of the anchovy, that is, the part between the resorption of the yolk-sac and the development of an inflated swimbladder. This is a critical phase when the larva switches from endogenous to exogenous feeding. Thus, its survival depends strongly on its ability to find and catch food while its movement in the water column is impaired by the lack of a functional swimbladder. Following the same approach as in a previous work by Arino et al. [1996], the state of the targeted population is determined, at every moment $t$, by two densities: one, $L_{e}$, is associated with demography while the other one, $W_{e}$, is more reflective of physiology. Competition, as well as density-dependence effects, have been considered negligible since, from field data, it seems clear that eggs and early larvae disperse soon after spawning (Motos et al. [1991]). So, the equation for the demography is linear. Nonlinear effects and probably also threshold effects are in action in the variation of weight. The nonlinearity chosen here is an attempt to account for part of these effects. The main goal of the work was to derive a general formula for the rate of recruitment in the late larval stage in terms of the population that has completed successfully the yolk-sac period. In this respect, formula (4.15) is the main result of the paper: its generality is broad enough to encompass two completely different examples, Example 1 concentrating on the role of temperature on the rate of recruitment, and Example 2 showing the possible role of spatial heterogeneity. The paper considers a relatively small segment of the life cycle of the anchovy, seemingly the most crucial part, since it corresponds to the passage from endogenous to exogenous feeding (Regner [1985]). A model of the earlier phase from 
the egg stage to the end of the yolk-sac period has been set up by Arino et al. [1999]. Combined with the result of this paper, it allows the computation of a rate of recruitment from the egg into the late larval stage. Finally, the rate of recruitment from the egg into the juvenile stage, of main interest for fishery management, entails the study of the late larval stage, until the beginning of filter feeding (Hunter and Dorr [1982]; Mullin [1993]). This will be considered in future work.

\section{APPENDIX}

1. Determination of the early juveniles flux. The total density entering the late larval stage during a period $\Delta t$ is

$$
\left[\int_{\Omega} L_{l}(t, P) d P\right] \Delta t
$$

What we have to do now is to evaluate the difference

$$
L_{\text {pot }}(t+\Delta t)-L_{\text {pot }}(t)
$$

which we divide into two parts

$$
L_{\mathrm{pot}}(t+\Delta t)-L_{\mathrm{pot}}(t)=\Delta^{(1)} L_{\mathrm{pot}}+\Delta^{(2)} L_{\mathrm{pot}}
$$

with

$$
\Delta^{(1)} L_{\mathrm{pot}}=\int_{\mathcal{A}_{\Omega}(t+\Delta t)}\left[L_{e}(a, t+\Delta t, P)-L_{e}(a, t, P)\right] d a d P
$$

and

$$
\Delta^{(2)} L_{\mathrm{pot}}=\int_{\mathcal{A}_{\Omega}(t+\Delta t)} L_{e}(a, t, P) d a d P-\int_{\mathcal{A}_{\Omega}(t)} L_{e}(a, t, P) d a d P .
$$

Under some differentiability assumptions on $L_{e}$ that we will not specify, we can write:

$$
\Delta^{(1)} L_{\mathrm{pot}}=\left[\int_{\mathcal{A}_{\Omega}(t)} \frac{\partial L_{e}}{\partial t}(a, t, P) d a d P\right] \Delta t+o(\Delta t) .
$$


Using equation (3.11), we can change $\left(\partial L_{e} / \partial t\right)$ to $-\left(\partial L_{e} / \partial a\right)-\vec{V}(t, P)$. $\vec{\nabla}_{P} L_{e}-\mu L_{e}$, which, if we omit the $o(\Delta t)$ quantity, yields (A.6)

$$
\begin{aligned}
\Delta^{(1)} L_{\mathrm{pot}}= & -\left[\int_{\mathcal{A}_{\Omega}(t)}\left[\frac{\partial L_{e}}{\partial a}(a, t, P)\right] d a d P\right] \Delta t \\
& -\left[\int_{\mathcal{A}_{\Omega}(t)}\left[\vec{V}(t, P) \cdot \vec{\nabla}_{P} L_{e}+\mu(a, t) L_{e}(a, t, P)\right] d a d P\right] \Delta t .
\end{aligned}
$$

Let us now consider $\Delta^{(2)} L_{\text {pot }}$. This quantity can be written in the form

$$
\begin{aligned}
\Delta^{(2)} L_{\text {pot }}=\int_{\mathcal{A}_{\Omega}(t+\Delta t) \backslash \mathcal{A}_{\Omega}(t)} & L_{e}(a, t, P) d a d P \\
& -\int_{\mathcal{A}_{\Omega}(t) \backslash \mathcal{A}_{\Omega}(t+\Delta t)} L_{e}(a, t, P) d a d P .
\end{aligned}
$$

Let us examine the integral over $\mathcal{A}_{\Omega}(t+\Delta t) \backslash \mathcal{A}_{\Omega}(t)$. The contributions of $L_{e}$ which survive for each $\Delta t>0$ small enough are those in the vicinity of the surface $\Sigma_{\Omega}(t)=\left\{(a, P) \in \overline{\mathcal{A}_{\Omega}(t)}: W_{e}(a, t, P)=w^{*}\right\}$ and such that $\left(\partial W_{e} / \partial t\right)(a, t, P)<0$. For any point $(a, P)$ in $\Sigma_{\Omega}(t)$, there is a small portion of the normal line to $\Sigma_{\Omega}(t)$ at $(a, P)$ which belongs to the set $\mathcal{A}_{\Omega}(t+\Delta t) \backslash \mathcal{A}_{\Omega}(t)$. An estimate of the length of this portion is

$$
\frac{\delta(a, t, P)}{\Delta t} \equiv \frac{\left(\partial W_{e} / \partial t\right)}{\left(\left|\left(\partial W_{e} / \partial a\right)\right|^{2}+\left|\nabla_{P} W_{e}\right|^{2}\right)^{1 / 2}}
$$

The same formula holds for $\mathcal{A}_{\Omega}(t) \backslash \mathcal{A}_{\Omega}(t+\Delta t)$, with $\delta>0$ this time. In terms of $\delta$, we have the following expression for $\Delta^{(2)} L_{\text {pot }}$

$$
\Delta^{(2)} L_{\mathrm{pot}}=-\int_{\Sigma_{\Omega}(t)} L_{e}(a, t, P) \delta(a, t, P) d \Sigma_{\Omega}(t)(a, P)
$$

where the notation $d \Sigma_{\Omega}(t)(a, P)$ stands for the surface integral over $\Sigma_{\Omega}(t)$, that is:

$$
d \Sigma_{\Omega}(t)(a, P)=\frac{\left(\left|\left(\partial W_{e} / \partial a\right)\right|^{2}+\left|\nabla_{P} W_{e}\right|^{2}\right)^{1 / 2}}{\left|\left(\partial W_{e} / \partial a\right)\right|} d P
$$


which yields

$$
\Delta^{(2)} L_{\mathrm{pot}}=-\int_{\Sigma_{\Omega_{\sigma}}(t)} L_{e}\left(a^{*}(t, P), t, P\right) \frac{\partial W_{e} / \partial t}{\left|\partial W_{e} / \partial a\right|} d P \Delta t .
$$

Coming back to the estimate of the variation of $L_{\text {pot }}$ within the time interval $[t, t+\Delta t]$ we can write, first of all

(A.10)

$$
\begin{aligned}
& L_{\mathrm{pot}}(t+\Delta t)-L_{\mathrm{pot}}(t) \\
& =\left[\int_{\Omega} L_{e}(0, t, P) d P\right] \Delta t-\left[\int_{\mathcal{A}_{\Omega}(t)} L_{e}(a, t, P) \mu(a, t) d a d P\right] \Delta t \\
& -\left[\int_{\partial \mathcal{A}_{\Omega \sigma}(t)}\left[\int_{\{a:(a, P) \in \overline{\mathcal{A}(t)}\}} L_{e}(a, t, P) d a\right] \vec{V}(t, P) \cdot \vec{n}(P) \sigma(d P)\right] \Delta t \\
& -\left[\int_{\Omega} L_{l}(t, P) d P\right] \Delta t
\end{aligned}
$$

The above expression is to be balanced with the one in terms of $\Delta^{(1)} L_{\text {pot }}$ (A.6) and $\Delta^{(2)} L_{\text {pot }}$ (A.9), that is,

$$
\begin{aligned}
& J_{\mathrm{pot}}(t+\Delta t)-J_{\mathrm{pot}}(t) \\
& =-\left[\int_{\mathcal{A}_{\Omega}(t)}\left[\frac{\partial L}{\partial a}(a, t, P)+\vec{V}(t, P) \cdot \vec{\nabla}_{P} L+\mu(a, t) L(a, t, P)\right] d a d P\right] \Delta t \\
& \quad-\left[\int_{\Sigma_{\Omega_{\sigma}}(t)} L\left(a^{*}(t, P), t, P\right) \frac{\partial W / \partial t}{|\partial W / \partial a|} d P\right] \Delta t .
\end{aligned}
$$

In the expression (A.10), we note that

$$
\begin{aligned}
\int_{\partial \mathcal{A}_{\Omega \sigma}(t)} & {\left[\int_{\{a:(a, P) \in \overline{\mathcal{A}(t)\}}} L_{e}(a, t, P) d a\right] \vec{V}(t, P) \cdot \vec{n}(P) \sigma(d P) } \\
= & \int_{\mathcal{A}_{\Omega \sigma}(t)} \operatorname{div}_{P}\left\{\left[\int_{\{a:(a, P) \in \overline{\mathcal{A}(t)}\}} L_{e}(a, t, P) d a\right] \vec{V}(t, P)\right\} d P .
\end{aligned}
$$

The integral over $\mathcal{A}_{\Omega \sigma}(t)$ breaks down into the sum of two quantities:

$$
\begin{array}{r}
\int_{\mathcal{A}_{\Omega \sigma}(t)}\left[\int \vec{\nabla}_{P} 1_{\{a:(a, P) \in \overline{\mathcal{A}(t)\}}}(a) L_{e}(a, t, P) d a\right] \cdot \vec{V}(t, P) d P \\
+\int_{\mathcal{A}_{\Omega}(t)} \vec{\nabla}_{P} L_{e}(a, t, P) \cdot \vec{V}(t, P) d P,
\end{array}
$$


where, for any subset $S$, we denote $1_{S}$ the indicator function of $S$. After elimination of the quantities which appear in both expressions (A.10) and (A.11), and division by $\Delta t$, we arrive at a first relationship:

$$
\begin{aligned}
& \int_{\Omega} L_{e}(0, t, P) d P-\int_{\Omega} L_{l}(t, P) d P \\
& \quad-\int_{\mathcal{A}_{\Omega \sigma}(t)}\left[\int \vec{\nabla}_{P} 1_{\{a:(a, P) \in \overline{\mathcal{A}(t)\}}}(a) L_{e}(a, t, P) d a\right] \cdot \vec{V}(t, P) d P \\
& =-\int_{\mathcal{A}_{\Omega}(t)} \frac{\partial L_{e}}{\partial a}(a, t, P) d a d P-\int_{\Sigma_{\Omega_{\sigma}}(t)} L_{e}\left(a^{*}(t, P), t, P\right) \frac{\left(\partial W_{e} / \partial t\right)}{\mid\left(\partial W_{e} / \partial a \mid\right)} d P .
\end{aligned}
$$

From the definition of $\mathcal{A}_{\Omega}(t)$ and the observation made above that $a^{*}(t, P)$ is, for each $P$, the lowest upper bound of the $a$ such that $(a, P) \in \mathcal{A}_{\Omega}(t)$, we deduce that

$$
\begin{aligned}
& \int_{\mathcal{A}_{\Omega}(t)} \frac{\partial L_{e}}{\partial a}(a, t, P) d a d P \\
& \quad=\int_{\mathcal{A}_{\Omega \sigma}(t)} L_{e}\left(a^{*}(t, P), t, P\right) d P-\int_{\Omega} L_{e}(0, t, P) d P
\end{aligned}
$$

Substituting the righthand side of (5.13) in (5.12), we obtain, after elimination of $\int_{\Omega} L_{e}(0, t, P) d P$,

$$
\begin{aligned}
& \int_{\Omega} L_{l}(t, P) d P \\
& =-\int_{\mathcal{A}_{\Omega \sigma}(t)}\left[\int \vec{\nabla}_{P} 1_{\{a:(a, P) \in \overline{\mathcal{A}(t)}\}}(a) L_{e}(a, t, P) d a\right] d \cdot \vec{V}(t, P) d P \\
& \quad+\int_{\mathcal{A}_{\Omega \sigma}(t)} L_{e}\left(a^{*}(t, P), t, P\right) d P \\
& \quad+\int_{\Sigma_{\Omega_{\sigma}}(t)} L_{e}\left(a^{*}(t, P), t, P\right) \frac{\left(\partial W_{e} / \partial t\right)}{\left|\left(\partial W_{e} / \partial a\right)\right|} d P .
\end{aligned}
$$

This equality is true for each open subset $\Omega$ allows to deduce equality of the functions under the integrals. We obtain a general formula for 
the flux of early juveniles

$$
\begin{aligned}
L_{l}(t, P)= & 1_{\mathcal{A}_{\sigma}(t)}(P) L_{e}\left(a^{*}(t, P), t, P\right)-1_{\mathcal{A}_{\sigma}(t)}(P) \\
& \cdot\left[\int \vec{\nabla}_{P} 1_{\{a:(a, P) \in \overline{\mathcal{A}(t)\}}}(a) L_{e}(a, t, P) d a\right] \cdot \vec{V}(t, P) \\
& +1_{\Sigma_{\sigma}(t)}(P) L_{e}\left(a^{*}(t, P), t, P\right) \frac{\left(\partial W_{e} / \partial t\right)}{\left|\left(\partial W_{e} / \partial a\right)\right|}
\end{aligned}
$$

2. Example 2. We do this and we keep the same notations for the integrated state variables. In spite of all the above simplifying assumptions, the problem at hand is not trivial. The main difficulty is in describing the admissible set $\mathcal{A}(t)$, since this time the weight $W_{e}$ is not always increasing with respect to age. In order to determine $\mathcal{A}(t)$, as given by formula (4.10), we first have to compute the flow $\Phi=\Phi\left(s, t_{0}, P_{0}\right)=(t(s), x(s))$. The derivation is straightforward

$$
t(s)=s+t_{0}, x(s)=s U_{1}+x_{0} .
$$

It is also very easy to solve equation (4.20). We arrive at the following formula

$$
\begin{aligned}
W_{e}(a, t, x)= & \exp (-\alpha a) W_{0} \\
& +\int_{t-a}^{t} \exp [-\alpha(t-\sigma)] k(\sigma) f(\sigma) \varphi\left(\sigma, x+(\sigma-t) U_{1}\right) d \sigma
\end{aligned}
$$

where, substituting for $\varphi$ its expression in terms of $\varphi_{0}$ and $\widetilde{\varphi}$ obtained by solving equation (4.19), that is,

$$
\begin{aligned}
& \varphi(t, x)=\exp (C(\bar{N}) t) \varphi_{0}\left(x-U_{1} t\right), \quad \text { for } x-U_{1} t<\xi \\
& \varphi(t, x)=\exp \left(C(\bar{N}) \frac{x-\xi}{U_{1}}\right) \widetilde{\varphi}\left(t-\frac{x-\xi}{U_{1}}\right), \quad \text { for } x-U_{1} t>\xi
\end{aligned}
$$


gives

$$
\begin{aligned}
& W_{e}(a, t, x)=\exp (-\alpha a) W_{0}+\varphi_{0}\left(x-t U_{1}\right) \\
& \cdot \exp (-\alpha t) \int_{t-a}^{t} \exp [(\alpha+C(\bar{N})) \sigma] k(\sigma) f(\sigma) d \sigma \\
& \text { if } x-U_{1} t<\xi \text {, } \\
& W_{e}(a, t, x)=\exp (-\alpha a) W_{0}+\widetilde{\varphi}\left(t-\frac{x-\xi}{U_{1}}\right) \\
& \cdot \exp \left(-\alpha t+C(\bar{N}) \frac{x-\xi}{U_{1}}\right) \int_{t-a}^{t} \exp [\alpha \sigma] k(\sigma) f(\sigma) \\
& \text { if } x-U_{1} t>\xi \text {. }
\end{aligned}
$$

To make the above formula more explicit, we further assume that the product

$$
k(t) f(t)=\kappa
$$

is a constant. This leads to

$$
\begin{aligned}
W_{e}(a, t, x)= & \exp (-\alpha a) W_{0}+\kappa \varphi_{0}\left(x-t U_{1}\right) \\
& \cdot \exp [C(\bar{N}) t] \frac{[1-\exp -(\alpha+C(\bar{N})) a]}{(\alpha+C(\bar{N}))} \\
& \text { if } x-U_{1} t<\xi \\
W_{e}(a, t, x)= & \exp (-\alpha a) W_{0}+\kappa \widetilde{\varphi}\left(t-\frac{x-\xi}{U_{1}}\right) \\
& \cdot \exp \left(C(\bar{N}) \frac{x-\xi}{U_{1}}\right) \frac{[1-\exp -\alpha a]}{\alpha} \\
& \text { if } x-U_{1} t>\xi .
\end{aligned}
$$

We will now restrict our attention to an even more simplified situation which, however, is feasible: we assume that

$$
\varphi_{0}=0 \text { and } \widetilde{\varphi} \text { is constant, } \quad \widetilde{\varphi}=\widetilde{\varphi}_{C} .
$$

This would correspond to the case of phytoplankton coming into a region from an external source. The formulae for $W$ reduces to the 
following

$$
\begin{aligned}
W_{e}(a, t, x)= & \exp (-\alpha a) W_{0} \quad \text { if } x-U_{1} t<\xi \\
W_{e}(a, t, x)= & \exp (-\alpha a) W_{0} \\
& +\kappa \widetilde{\varphi}_{C} \exp \left(C(\bar{N}) \frac{x-\xi}{U_{1}}\right) \frac{[1-\exp -\alpha a]}{\alpha} \\
& \text { if } x-U_{1} t>\xi .
\end{aligned}
$$

The restriction $\bar{W}_{e}(s)$ of $W_{e}$ along a characteristic line $t(s)=s+t_{0}$, $x(s)=s U_{1}+x_{0}$, starting from a point $\left(0, t_{0}, x_{0}\right)$ gives

$$
\begin{aligned}
\bar{W}_{e}(s)= & \exp (-\alpha s) W_{0} \quad \text { if } x_{0}-U_{1} t_{0}<\xi, \\
\bar{W}_{e}(s)= & \exp (-\alpha s) W_{0} \\
& +\kappa \widetilde{\varphi}_{C} \exp \left(C(\bar{N})\left[s+\frac{x_{0}-\xi}{U_{1}}\right]\right) \frac{[1-\exp -\alpha s]}{\alpha} \\
& \text { if } x_{0}-U_{1} t_{0}>\xi .
\end{aligned}
$$

Formula (A.19) shows that some initial values $\left(0, t_{0}, x_{0}\right)$ will automatically drive the larvae to starvation and death: this is when $x_{0}-U_{1} t_{0}<\xi$. In contrast, in the complementary subset, we may have, according to the parameters and the initial value, either initial weight loss followed by gain, or gain from the beginning. We will continue our computations under the following additional assumption

$$
-\alpha W_{0}+\kappa \widetilde{\varphi}_{C}>0
$$

a situation when the weight increases from the beginning in the set

$$
\left\{\left(t_{0}, x_{0}\right): x_{0}-U_{1} t_{0}>\xi\right\} .
$$

In order to compute the recruitment rate in the late larval stage, we first have to determine the set $\mathcal{A}(t)$. It is made up of two subsets: 1$)$ for those points $(a, t, x)$ for which $x-U_{1} t<\xi$, it is defined by the condition $\exp (-\alpha a) W_{0}>w_{\min }$. We will denote $\mathcal{A}^{(1)}(t)$ this set. Thus, we have

$$
\left.\mathcal{A}^{(1)}(t)=\right] 0, \frac{1}{\alpha} \ln \left(\frac{W_{0}}{w_{\min }}\right)\left[\times\left\{(t, x): x-U_{1} t<\xi\right\} .\right.
$$


2 ) For the points $(a, t, x)$ for which $x-U_{1} t>\xi$, the condition on $a$ is just

$$
\exp (-\alpha a) W_{0}+\kappa \widetilde{\varphi}_{C} \exp \left(C(\bar{N}) \frac{x-\xi}{U_{1}}\right) \frac{[1-\exp -\alpha a]}{\alpha}<w^{*},
$$

which is equivalent to

$$
a<\frac{1}{\alpha} \ln \left[\frac{\kappa \widetilde{\varphi}_{C} \exp \left(C(\bar{N})\left(x-\xi / U_{1}\right)-\alpha W_{0}\right)}{\kappa \widetilde{\varphi}_{C} \exp \left(C(\bar{N})\left(x-\xi / U_{1}\right)-\alpha w^{*}\right.}\right] \equiv a^{(2)}(x) .
$$

It should be noted that formula (A.22) is not always defined. While condition (A.20) ensures that $\kappa \widetilde{\varphi}_{C} \exp \left(C(\bar{N})\left(x-\xi / U_{1}\right)\right)-\alpha W_{0}>0$, no assumption has been made regarding the quantity $\alpha w^{*}$ compared to $\kappa \widetilde{\varphi}_{C}$. If $\alpha w^{*}>\kappa \widetilde{\varphi}_{C}$ for some values of $x$, the inequality defining $a^{(2)}(x)$ holds true for all $a$, which implies that $a^{(2)}(x)=+\infty$, in this case, meaning that the feeding rate is too low to allow the larva to reach the threshold $w^{*}$ in finite time. So, for definiteness, we will extend $a^{(2)}(x)$ to the set of $x$ such that $\kappa \widetilde{\varphi}_{C} \exp \left(C(\bar{N})\left(x-\xi / U_{1}\right)\right)-\alpha w^{*}<0$ by setting $a^{(2)}(x)=+\infty$ on this set. The corresponding subset of $\mathcal{A}(t)$, denoted $\mathcal{A}^{(2)}(t)$, is defined by

$$
\mathcal{A}^{(2)}(t)=\left\{(a, t, x): x-U_{1} t>\xi \text { and } a<a^{(2)}(x)\right\}
$$

where $a^{(2)}(x)$ is given by formula $\left(\right.$ A.22) or $a^{(2)}(x)=+\infty$.

Finally, we have

$$
\mathcal{A}(t)=\mathcal{A}^{(1)}(t) \cup \mathcal{A}^{(2)}(t)
$$

from which we deduce that

$$
\mathcal{A}_{\sigma}(t)=D
$$

and

$$
\Sigma(t)=\left\{\left(a^{(2)}(x), x\right): x \geq U_{1} t+\xi \text { and } a^{(2)}(x)<+\infty\right\}
$$

which yields

$$
\Sigma_{\sigma}(t)=\left\{x: x \geq U_{1} t+\xi\right\} \cap \overline{\left\{x: a^{(2)}(x)<+\infty\right\}} .
$$

For $x \in \Sigma_{\sigma}(t)$, we have

$$
\frac{\partial W_{e}}{\partial t}=0
$$


and

$$
\frac{\partial W_{e}}{\partial a}=\exp (-\alpha a)\left[-\alpha W_{0}+\kappa \widetilde{\varphi}_{C} \exp \left(C(\bar{N}) \frac{x-\xi}{U_{1}}\right)\right]
$$

We are now ready to compute $L_{l}(t, x)$ as given by formula (A.14). Noting first that the function $a^{*}(t, x)$ has $\mathcal{A}_{\sigma}^{(2)}(t)$ as a domain and is such that $a^{*}(t, x)=a^{(2)}(x)$, formula (A.14) reduces to

$$
L_{l}(t, x)=\left(\left[1-U_{1} a^{(2) \prime}(x)\right]+1_{\Sigma_{\sigma}(t)}(x) \frac{\partial W_{e} / \partial t}{\left|\partial W_{e} / \partial a\right|}\right) L_{e}\left(a^{(2)}(x), t, x\right) .
$$

Combining the above results, and using notably formula (5.42), it follows that

$$
\begin{array}{ll}
L_{l}(t, x)=\left[1-U_{1} a^{(2) \prime}(x)\right] L_{e}\left(a^{(2)}(x), t, x\right) \\
& \text { if } x>U_{1} t+\xi \text { and } a^{(2)}(x)<+\infty, \\
L_{l}(t, x)=0 & \text { otherwise. }
\end{array}
$$

Acknowledgments. The authors are pleased to thank the referees for their valuable comments.

\section{REFERENCES}

O. Arino, C. Koutsikopoulos and A. Ramzi [1996], Elements of Mathematical Modeling of Evolution in Number of a Sole Population, J. Biol. Systems 4, 445-458.

O. Arino, A. Boussouar and P. Prouzet [1999], Modeling of the Larval Stage of the Anchovy of the Bay of Biscay. Estimation of the Rate of Recruitment in the Juvenile Stage, Project 96/048 DG XIV, Final Report, in preparation.

A. Borja, A. Uriarte, V. Valencia, L. Motos and A. Uriarte [1996], Relationships between Anchovy (Engraulis encrasicolus L.) Recruitment and the Environment in the Bay of Biscay, Scientia Marina 60 (supl. 2), 179-192.

J. Csirke [1995], Fluctuations in Abundance of Small and Mid-Size Pelagics, Scientia Marina 59, 481-492.

D.H. Cushing [1990], Plankton Production and Year Class Strength in Fish Populations: An Update of Match/Mismatch Hypothesis, Adv. Mar. Biol. 26, 249-293.

J.R. Hunter and H. Dorr [1982], Thresholds for Filter Feeding in Northern Anchovy, Engraulis Mordax, CalCOFI Report, Vol. 23.

A.D. Jassby and T. Platt [1976], Mathematical Formulation of the Relationship between Photosynthesis and Light for Phytoplankton, Limnol. Oceanogr. 21, $540-547$. 
W.C. Leggett and E. Deblois [1994], Recruitment in Marine Fishes: Is It Regulated by Starvation and Predation in the Egg and Larval Stages?, Netherlands J. Sea Research 32, 119-134.

J.J. MacIsaac and R.C. Dugdale [1969], The Kinetics of Nitrate and Ammonium Uptake by Natural Populations of Marine Phytoplankton, Deep-Sea Res. 16, 45-57.

L. Motos, J. Santiago and A. Uriarte [1991], Assessment of Spawning Biomass of the Bay of Biscay Anchovy Population, Engraulis Encrasicolus L., Using the Daily Egg Production Method, ICES CM/H:33.

M.M. Mullin [1993], Webs and Scales, Physical and Ecological Processes in Marine Fish Recruitment, University of Washington Press, Seattle, Washington.

O. Pardo [2000], Global Stability for a Phytoplankton-Nutrient System, J. Biol. System 8, 195-209.

R.M. Peterman, M.J. Bradford, N.C.H. Lo and R.D. Methot [1988], Contribution of Early Life Stages to Interannual Variability in Recruitment of Northern Anchovy (Engraulis Mordax), Canad. J. Fish. Aquat. Sci. 45, 8-16.

S. Pond and G.L. Pickard [1983], Introductory Dynamical Oceanography, 2nd ed., Pergamon Press, New York.

P. Re [1994], Anchovy Spawning in Mira Estuary (1985/1992), Nova serie 27, 423-454.

S. Regner [1985], Ecology of Planktonic Stages of the Anchovy, Acta Adriat. 26, $5-113$.

A.H. Taylor, J.R.W. Harris and J. Aiken [1986], The Interaction of Physical and Biological Process in a Model of the Vertical Distribution of Phytoplankton under Stratification, in Marine Interfaces Ecohydrodynamics (Jacques C. Nihoul, ed.), Elsevier Oceanography Series, 42, New York, 313-330.

A. Uriarte, P. Prouzet and B. Villamor [1996], Bay of Biscay and Ilbero Anchovy Populations and Their Fisheries, Scientia Marina 60 (supl. 2), 237-255. 